\title{
No Added Sugar Mixed Fruit Prebiotic Jam
}

\author{
S Ramya $^{1 *}$, J.Poongothai ${ }^{1}$ \\ \{sr.bio@psgtech.ac.in ${ }^{1 *}$,jpi.bio@psgtech.ac.in $\left.{ }^{1}\right\}$ \\ Department of Bio Technology, PSG College of Technology, Coimbatore, TamilNadu, India ${ }^{1}$
}

\begin{abstract}
Human gut is occupied by thousands of microorganisms called probiotics which enhances the host health by multiple ways. Some of the health benefits of probiotics are increase in availability of vitamins, boosts immune system, better digestion and so on. Considering the importance of probiotics many functional foods with probiotic supplement are developed and available in the market. However it is tremendously difficult to develop a probiotic based food because it takes several steps and techniques during processing; there is a question of which strain to choose and how much strain should be added. This paves way for development of prebiotic foods. Prebiotics are non-digestible carbohydrate that are digested by gut microbes and thus helps in the survival of probiotics. So prebiotics are in spotlight. Indian plant materials contain prebiotics in nature. In this study we are focussing on preparation of sugar-free prebiotic jam solely prepared from fruits available in India. Optimisation of ingredients was done using design expert software and hedonic scale testing was used for sensory evaluation.
\end{abstract}

Keywords: Design expert software, functional foods, gut microbes, Indian plant material,prebiotics, prebiotic jam, probiotics.

\section{Introduction}

Functional foods are product that resemble traditional foods but possesses additional health benefits than basic nutrition. Gut microbiota known as probiotics are naturally present in human gastrointestinal tract. These microbes are present in stomach, small intestine and large intestine however majority of these are present in large intestine. Human diet plays a major role in growth of microorganisms. In particular non digestible carbohydrates called prebiotics is fermented by probiotics and acts as source of growth. This is done by degrading indigestible binds of prebiotics. However synbiotics are product that uses both probiotics and prebiotics. A mixture of probiotic live bacteria and required amount of prebiotic when utilised together are defined as "Synbiotics", thus probiotics can survive to its fullest with the presence of prebiotics [1]

Among many probiotics, Lactobacillus is the widely used microorganism. However, currently Bifidobacterium is encouragingly used and considered as a potential probiotic since Bifidobacterium species belong to dominant anaerobic flora of the colon. Some of the challenges in developing a probiotic food are strain selection, its biological properties, addition level, toxicity, identification and enumeration of viable strains and stability during storage. Since the use of probiotics in food involve several crucial and determining steps, addition of prebiotics into the food decreases the risk, make procedure easier and also possess health benefits by potentially increasing the gut microbiota. The most common prebiotics are 
Fructooligosaccharides (FOS), Galactooligosaccharides(GOS), Trans-galactooligosaccharides (TOS). In order to be utilized by the indigenous microflora in the large intestine, prebiotics must overcome two barriers. One is to withstand acid hydrolysis in the stomach and it must not be absorbed in the small intestine[2].

Prebiotic can be obtained by two ways

1. Naturally present in plants such as banana, chicory etc.,

2. Synthesized from enzymatically degrading polysaccharides.

Majority of the study on sources of prebiotics are western origin hence Indian fruits and vegetables need to be given attention. Fruits and vegetables of Indian origin are proved to have array of phytochemicals which have health benefits individually or in combination [3].ndian fruits and vegetables must be given attention in order to develop prebiotic functional food products with cheap, easy and effective manufacturing process.

Sensory analysis represents a decisive step during the various stages of food product development. In case of obtaining sensory information that is well enough to be accepted in consumer's market is achieved by proper techniques for probiotics, prebiotics and synbiotics showing continuous improvement in functional food category[4].In order to attain its commercial value any food must have sensory characteristics well accepted by consumer market, safety characteristics for consumption and nutritional requirements.

An adequate and efficient sensory methodology will provide a prior knowledge on its acceptance on consumer market. This serves as a basis for making alterations. However parallel comparing with similar commercial product is necessary. The products in which prebiotics and probiotics are added have shown considerably different flavour profile compare to the conventional food; however the sensory impact of these functional ingredients has not been studied in detail [5].

Discriminative, affective and descriptive are the three types of sensory test that will be done for food products. Discriminative test gives limited information. This tests clarifies if there are any differences between functional (prebiotic, probiotic or symbiotic) food product and the conventional one. Affective sensory tests allows one to gain knowledge on the consumer's evaluation on quantity of the product and performance among functional and conventional food. Whereas descriptive test develops a complete sensory profile of the product.

Design Expert software for design of experiments (DOE) serves many functions such as screening, modelling and optimisation. In order to work with the software some questions need to be answered.

1. What is the objective of the study?

2. Which factors are to be examined?

3. What are the responses that need to be measured?

4. Does the budget allow number of runs acquired?

Rows and columns in the design define the framework of the experiment. The rows represent runs while the columns contain design information, components, factors, and responses. There are four main types of design methods.

1. Response surface

2. Factorial

3. Mixture

4. Custom

Considering the importance of prebiotic functional foods and also consumer's focus on healthy diet, this study aims on productizing no added mixed fruit prebiotic jam which solely made of fruits available in India. It is also to be noted that fruits used are rich sources of 
prebiotics. In order to reach best sensory requirements, Central composite design has been used to perform sensory evaluation. Central Composite designs (CCD) are based on 2-level factorial designs, augmented with center and axial points to fit quadratic models. Categoric factors can be added to these designs, however, the design is duplicated for every categoric treatment combination.

\section{Materials and methods}

Mixed fruit jam has been prepared with no added sugar to it. Novelty of the study is that no prebiotic jam is yet in the market using Indian fruits. Jam can be consumed by people of all age and because of its sugar free nature it is diabetic friendly too.

\section{A. Materials}

Fruits (Apple, fig, Pomegranate, papaya, grapes), pectin, Citric acid, potassium sorbate, Fructo oligosaccharides (FOS).

\section{B. Methods:}

1) Selection of fruits It is well believed that prebiotics are naturally present in plant materials. Previous studies are focused on western origin plant materials such as artichoke, garlic, onion, chicory, inulin, asparagus and so on. It is equally important to know the significance and prebiotic potential of locally available as well as edible plant materials. Whole fruit approach allows making use of other functional properties of oligosaccharide rich fruits in spite of using pure oligosaccharide. It is essentialto make use of Indian fruits and vegetables as prebiotic food supplement.

Having the reference[6], in our research selected prebiotic potential fruits has been used in preparation of no added sugar mixed fruit prebiotic jam. The fruits used in our experiment are pomegranate, papaya, fig, grapes and apple. These fruits are selected based on their positive effect on sensory attribute of jam and some fruits are rejected because of seasonal availability.

\section{2) Calculation on amount of ingredient for preliminary studies}

Amount of fruits to be added are purely based on two main factors. First factor is prebiotic potential of fruits ie., based on the growth response of plant materials to probiotics (Yadav et al., 2014). The second factor is based on the effect of individual fruits to sensory characteristics of the jam. Pectin is used as a gelling agent. There are two types of pectin namely low methoxyl pectin and high methoxyl pectin. High methoxyl pectin is normally used in standard jam where sugar content will be high. Whereas low methoxyl pectin will be used in low calorie food product because it does not require sugar for its gelatin. In our preparation low methoxyl pectin is used since it is no added sugar jam. Potassium sorbate is used to inhibit molds and yeasts. Citric acid is used as a preservative. Fructo-oligosaccharide plays a major role as prebiotic.Amount of fruits added are listed in the table 2.

Table 1. Amount of fruits

\begin{tabular}{|l|l|l|}
\hline S.NO & FRUIT NAME & AMOUNT IN g \\
\hline 1 & Pomegranate & 30 \\
\hline 2 & Papaya & 30 \\
\hline
\end{tabular}




\begin{tabular}{|l|l|l|}
\hline 3 & Apple & 15 \\
\hline 4 & Grapes & 5 \\
\hline 5 & Fig & 5 \\
\hline & Total & 85 \\
\hline
\end{tabular}

Amount of ingredients to be added is determined by doing preliminary tests.

Table 2. Preliminary tests.

\begin{tabular}{|c|c|c|} 
Preliminary test & Ingredient & Amount in grams (g) \\
\hline \multirow{4}{*}{ Trial 1 } & Pectin & 0.925 \\
\cline { 2 - 3 } & Potassium sorbate & 0.135 \\
\cline { 2 - 3 } & Citric acid & 0.100 \\
\cline { 2 - 3 } & FOS & 12.000 \\
\hline \multirow{4}{*}{ Trial 2 } & Pectin & 0.925 \\
\cline { 2 - 3 } & Potassium sorbate & 0.135 \\
\cline { 2 - 3 } & Citric acid & 0.050 \\
\cline { 2 - 3 } & FOS & 12.000 \\
\hline
\end{tabular}

\section{3) Preparation of jam}

The fruits are washed with clean water and the pulp of the fruits is separated manually using knife. In case of pomegranate and grapes juice is extracted. The Fruit pulp and juice were weighed by using a weighing machine. Then the pulp was grinded well to attain homogenised mixture. The mixture was then transferred to a non-stick pan and made it to boil in low flame. When a boil is reached potassium sorbate, fructo oligosaccharide and low methoxyl pectin were added. The mixture is stirred continuously until all the ingredients mix well. Weighed citric acid crystals are diluted and made into liquid by adding tea spoon of water to it. Once the jam consistency is arrived citric acid liquid is added to it and mixed well. Jam is then transferred to a glass container and allowed to cool. It was then stored in refrigerator.

\section{4) Optimisation of ingredients}

Optimisation of ingredients for the main study was done by using design expert software. The need for optimisation is each ingredient used in the preparation of jam has its own ability to alter the sensory characteristics of the jam to a greater extent. For example larger amount of pectin influences the gelling property of the jam by making it thicker while pectin in very low amount does not results in jam consistency. Central composite design was used in the experiment for optimisation. There are different types in central composite design in which we chose small to obtain minimum runs.

Totally there are eight factors Viz pomegranate, papaya, grapes, fig, apple, pectin, potassium sorbate and citric acid. Values obtained from preliminary test 2 are taken as mid value. For all the fruits low and high value was taken as \pm 5 while for other ingredients it was taken as \pm 0.02 from mid value.

Fig 1. Central composite design input

\begin{tabular}{|l|l|l|l|l|l|}
\hline Name & Units & Low & High & $(-)$ alpha & $(+)$ alpha \\
\hline Pomogranate & $\mathrm{g}$ & 25 & 35 & 21.591 & 38.409 \\
\hline Papaya & $\mathrm{g}$ & 25 & 35 & 21.591 & 38.409 \\
\hline
\end{tabular}




\begin{tabular}{|l|l|l|l|l|l|} 
Apple & $\mathrm{g}$ & 10 & 20 & 6.59104 & 23.409 \\
\hline Grapes & $\mathrm{g}$ & 1 & 10 & -2.06807 & 13.0681 \\
\hline Fig & $\mathrm{g}$ & 1 & 10 & -2.06807 & 13.0681 \\
\hline Pectin & $\mathrm{g}$ & 0.905 & 0.945 & 0.891364 & 0.958636 \\
\hline CitricAcid & $\mathrm{g}$ & 0.03 & 0.07 & 0.016364 & 0.083636 \\
\hline $\begin{array}{l}\text { Potassium } \\
\text { sorbate }\end{array}$ & $\mathrm{g}$ & 0.115 & 0.155 & 0.101364 & 0.168636 \\
\hline
\end{tabular}

Fig 2. Central composite design output

\begin{tabular}{|l|l|l|l|l|l|}
\hline Name & Units & Low & High & $(-)$ alpha & (+)alpha \\
\hline Pomogranate & $\mathrm{g}$ & 25 & 35 & 21.591 & 38.409 \\
\hline Papaya & $\mathrm{g}$ & 25 & 35 & 21.591 & 38.409 \\
\hline Apple & $\mathrm{g}$ & 10 & 20 & 6.59104 & 23.409 \\
\hline Grapes & $\mathrm{g}$ & 1 & 10 & -2.06807 & 13.0681 \\
\hline Fig & $\mathrm{g}$ & 1 & 10 & -2.06807 & 13.0681 \\
\hline Pectin & $\mathrm{g}$ & 0.905 & 0.945 & 0.891364 & 0.958636 \\
\hline CitricAcid & $\mathrm{g}$ & 0.03 & 0.07 & 0.016364 & 0.083636 \\
\hline $\begin{array}{l}\text { Potassium } \\
\text { sorbate }\end{array}$ & $\mathrm{g}$ & 0.115 & 0.155 & 0.101364 & 0.168636 \\
\hline
\end{tabular}

Fig.3. Response input for central composite design

\begin{tabular}{|l|l|}
\hline Name & Units \\
\hline Taste & \\
\hline Texture & \\
\hline Aroma & \\
\hline
\end{tabular}

The final step is to give the response title. Response is the factors that are to be measured by doing the experiment. In this experiment response values are taste, texture and aroma.

\section{Results and discussion}

As mentioned before two preliminary tests were conducted to attain an approximate value for each ingredient to make Indian based prebiotic jam with no added sugar. Preliminary test 1 were conducted and accordingly preliminary test 2 were conducted with changes or alterations required in test 1 .

Observations on preliminary test 1 are as follows;

1. FOS was added at the last in test 1 and hence solubility of FOS powder was slow.

2. There was a colour change after addition of FOS.

3. Gelling property was observed after addition of low methoxyl pectin and jam consistency was obtained.

4. Addition of $0.1 \mathrm{~g}$ of citric acid caused sour taste.

Preliminary test 2 was done with little changes from test 1 . Observations on test 2 are as follows.

1. FOS was added along with pectin and the solubility was good. 
2. Amount of pectin, potassium sorbate and FOS were not altered.

3. Amount of citric acid was reduced to half and no sour taste was observed.

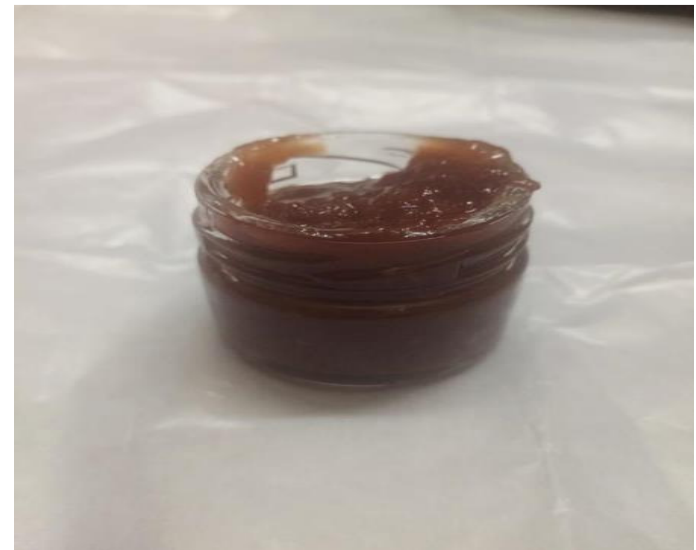

Fig.4 Prebiotic jam - Preliminary test 2 dated on 01/03/2021

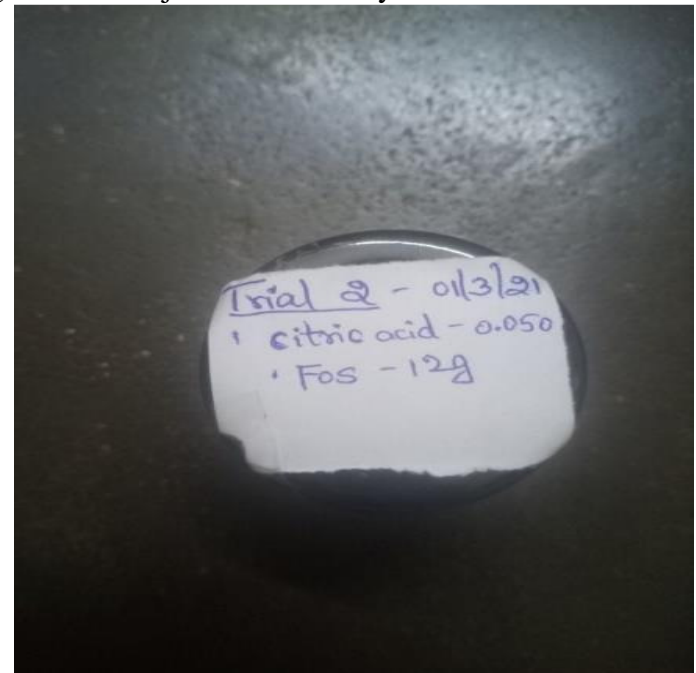

Fig.5 Label - Preliminary test 2

4. Jam was prepared on 1st march of 2021 and refrigerated. It was then observed on 18th april of 2021. The observations were

- Texture - There was no change in consistency of the product. There was no change in colour after storing for 48 days and also no fungal formation was observed.

- Taste - There was no change in taste. There was no reduction of sweetness and sour taste was not observed.

- Aroma - Aroma was same as before. Fruity odour was observed. No chemical odour was observed. 


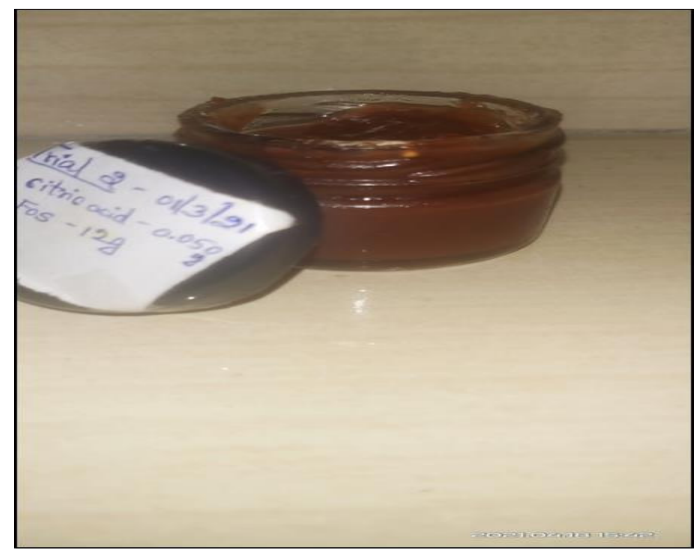

Fig.6 Prebiotic jam - observation of test 2 jam dated on 18/4/2021

\section{Future work}

Due to COVID 19 pandemic, we are not able to perform the study completely though the theory is described below. With the trial results obtained from Central composite design method, jam will be prepared. For each trail composition of the ingredients will vary and hence the sensory feature of jam. Therefore samples obtained from each trial will be given to human for sensory evaluation. This will be done by hedonic scale testing. After obtaining jam with best sensory characteristics, it has to be compared with standard jam in market. This is done by doing High pressure liquid chromatography which proves prebiotic potential of our product.

To prove the prebiotic potential and after effects of consuming this jam, animal study was needed. For animal study, 6 wistar rats are needed for the following purpose. Three rats will be used as control which will not be fed with prebiotic jam. These rats will undergo normal diet plan. Other three rats are fed with prebiotic jam along with the normal food. The amount of prebiotic jam would be $15 \%$ of total food consumption. The glycemic assay has to be performed through measurements of capillary glucose levels from the tail vein of each animal after $12 \mathrm{~h}$ of fasting. The main objective of the study is to show difference in gut microbiota (probiotics) level between the two groups. To achieve this fecal samples of the models have to be collected every 3 days and sequencing will be done to prove increase in probiotic level in host due to consumption of jam.

\section{Conclusion}

Jam was prepared on 1st march of 2021 and refrigerated. It was then observed on 18th april of 2021. The observations were

- Texture - There was no change in consistency of the product. There was no change in colour after storing for 48 days and also no fungal formation was observed. 
- Taste - There was no change in taste. There was no reduction of sweetness and sour taste was not observed.

- Aroma - Aroma was same as before. Fruity odour was observed. No chemical odour was observed.

Considering the importance of prebiotics in human health, prebiotic properties of Indian fruits and role of sensory characteristics in product development, the jam was prepared and further continuation of the study may lead to successful product in the market place.

\section{References}

[1] Panesar, P.S., Kaur, G., Panesar, R. and Bera, M.B., 2009. Synbiotics: potential dietary supplements in functional foods. IFIS: Berkshire, UK.

[2] Gibson, G.R., Scott, K.P., Rastall, R.A., Tuohy, K.M., Hotchkiss, A., Dubert-Ferrandon, A., Gareau, M., Murphy, E.F., Saulnier, D., Loh, G. and Macfarlane, S., 2010. Dietary prebiotics: current status and new definition. Food SciTechnol Bull Funct Foods, 7(1), pp.1-19.

[3] Rechkemmer, G., 2001. FunktionelleLebensmittel-Zukunft der ErnahrungoderMarketingStrategie?.MolkereiZeitung Welt Der Milch, 55(12/13), pp.S-332.

[4] Cruz, A.G., Cadena, R.S., Walter, E.H., Mortazavian, A.M., Granato, D., Faria, J.A. and Bolini, H.M., 2010. Sensory analysis: relevance for prebiotic, probiotic, and synbiotic product development. Comprehensive reviews in food science and food safety, 9(4), pp.358-373.

[5] Mattila-Sandholm, T., Blum, S., Collins, J.K., Crittenden, R., de Vos, W., Dunne, C., Fondén, R., Grenov, G., Isolauri, E., Kiely, B. and Marteau, P., 1999. Probiotics: towards demonstrating efficacy. Trends in Food Science \& Technology, 10(12), pp.393-399.

[6] Yadav, S.A., Gite, S.S., Lanjekar, V.B., Nilegaonkar, S.S. and Agte, V.V., 2014.In vitro screening of indigenous plant materials for prebiotic potential.International Journal of Current Microbiology and Applied Science, 3(11), pp.137-150. 